\section{$+\infty$

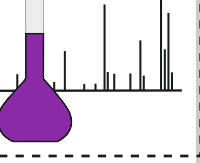 \\ Revealing Pre-analytical Pitfalls in Concentration Determination of Peptides by Quantification of Amino Acid Fluorescence}

Martina D. Allenspach, Jens A. Fuchs, and Christian Steuer*

${ }^{\star}$ Correspondence: Dr. C. Steuer, Department of Chemistry and Applied Biosciences, Institute of Pharmaceutical Sciences, Swiss Federal Institute of Technology (ETH) Vladimir-Prelog-Weg 4, CH-8093 Zürich, E-mail: christian.steuer@pharma.ethz.ch

Keywords: Antioxidant · Fluorescence · HPLC · Method development $\cdot$ Peptides · Validation

Synthetic peptides are important for drug discovery and have been identified as hit and lead compounds. There is a growing interest in developing simple, fast and accurate analytical assays for the quantification of peptides and proteins in the early drug discovery process. Most peptides are purified by reversed phase (RP)-HPLC by using an acidic modifier such as formic acid, acetic acid or trifluoroacetic acid (TFA). The peptides form salts with the acidic modifier, which binds specifically to free $\mathrm{NH}_{2}$ termini and to the side chains of exposed basic residues. Given the molecular weight of the TFA molecule (114 Da), the association of a single molecule to a peptide of $500 \mathrm{Da}$ would increase the formula weight by more than $20 \%$. The evaluation of the concentration of the peptides prior to biological activity testing is therefore crucial to avoid a source of error in concentrationdependent biological activity assays. However, the question is which molecular weight - either of the formed salts resulting from the purification process or the neutral form - is used for the calculation. Furthermore, other pre-analytical pitfalls such as weighing errors, pipetting performance or solubility issues can lead to false interpretation of the activity data. An analytical approach which allows to identify pre-analytical differences between the nominal and actual concentration is needed.

Therefore an easy, accurate and broadly applicable HPLCfluorescence-detection method for the quantitative determination of the aromatic amino acids (AAA) tyrosine (Tyr), phenylalanine (Phe) and tryptophan (Trp) using an isocratic elution was developed and validated. The peptides are hydrolyzed under heated and acidic conditions to yield the monomeric AAAs. To prevent the AAAs from oxidative decomposition, cysteine as protective agent is applied. The separation of the three AAAs and the internal standard was performed using ion-pair chromatography. The AAAs and the internal standard anthranilic acid are clearly separated. The power of the method was confirmed by the correct quantification of a protein reference standard to $98.6 \%$ over all fluorescence traces.

Interestingly, for a peptide which contains five basic AAs, the method yielded an $85 \%$ lower concentration than expected. The observed value was confirmed independently on Tyr and Trp traces. Considering five TFA counter ions, one would expect only a $40 \%$ lower amount of the indicated concentration. We speculate that the observed lower amount resulted from other impurities like fluorescent or UV-inactive organic species or residual solvents. The presented method requires only a few pre-analytical steps and can be implemented with standard laboratory equipment. Furthermore, only one solution is needed to perform quantification, UV-purity tests, and subsequent activity testing.

The developed fluorescence-based method is able to quantify peptides and proteins and is applied to determine accurately the concentration of peptides to limit the risk of erroneous activity data in drug discovery projects.

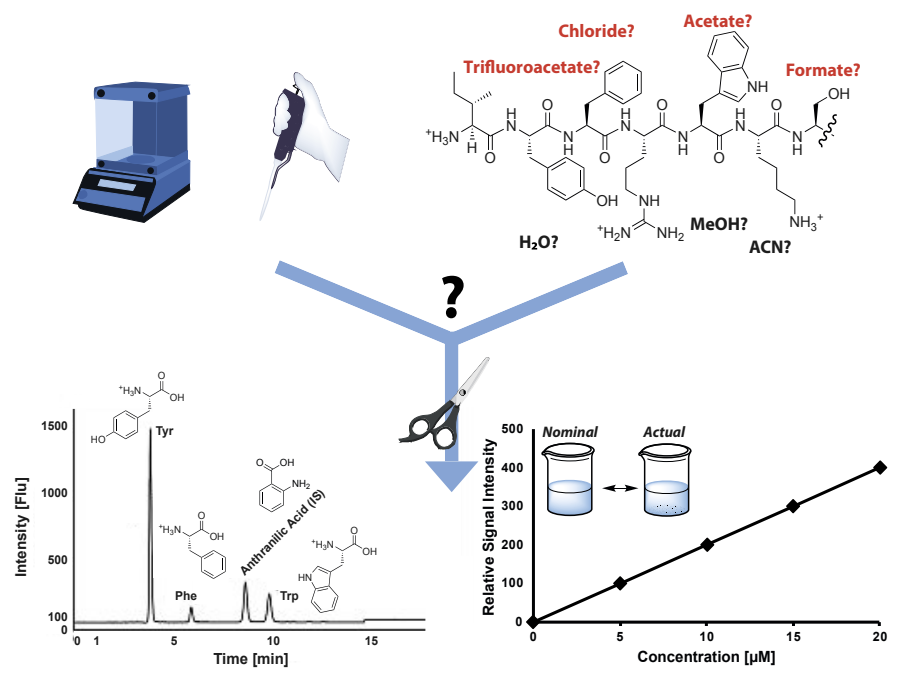

Salt formation, weighing errors, pipetting errors or solubility issues can lead to false declaration of peptide concentration. After chemical cleavage, the actual concentration of peptides can be determined by quantification of amino acid fluorescence.

Reference

M. D. Allenspach, J. A. Fuchs, N. Doriot, J. A. Hiss, G. Schneider, C. Steuer, J. Pept. Sci. 2018, 24, e3113. 